\section{P203 LUNG TRANSPLANTATION AND SURVIVAL IN IDIOPATHIC PULMONARY FIBROSIS - AN IRISH PERSPECTIVE}

P Riddell, I Lawrie, S Winward, K Redmond, JJ Egan; Mater Misericordiae University Hospital, Dublin, Ireland

\subsection{6/thoraxjnl-2013-204457.355}

Introduction and Objectives Idiopathic pulmonary fibrosis (IPF) is a chronic, fibrosing interstitial pneumonia of unknown aetiology. Its clinical course is unpredictable but invariably leads to progressive respiratory failure and death. Median survival from time of diagnosis, without transplant, is $2-3$ years.

IPF patients referred for transplantation often suffer from poor outcomes, due to organ shortage and rapid disease progression. In America, the introduction of the lung allocation score has greatly benefited the IPF cohort. Furthermore, an increasing proportion of these transplants have occurred in patients over the age of 65 - an age group that had previously been associated with high post-transplant mortality. Recent data has however contradicted this, showing that surgical outcomes and survival are satisfactory in this age group.

In a condition with no disease modifying medical therapy, we reviewed the survival benefit of lung transplantation in age-stratified groups to see if our data matched those seen internationally and to encourage further transplantation in this older population. Methods All patients with IPF who received lung transplantation in Ireland, since the beginning of the transplant service in 2005, were included $(n=30)$. Data collected included patient demographics, lung function, transplant details and survival data. Survival data was compared with IPF patients who had died while awaiting a transplant over the past 3 years $(n=20)$.

Results For those patients on the lung transplant waiting list, who did not receive a transplant, survival was unfortunately poor $(75 \%$ at 6 months, $30 \%$ at 12 months, and $15 \%$ at 18 months). However, following transplantation, all-age survival was $96.6 \%$ at 1 year, $90.1 \%$ at 2 years and $78.9 \%$ at 5 years. The 5 year survival for those transplanted over the age of 65 was $88.9 \%(\mathrm{n}=9)$.

\section{Survival: IPF patients on the lung transplant waiting list.}

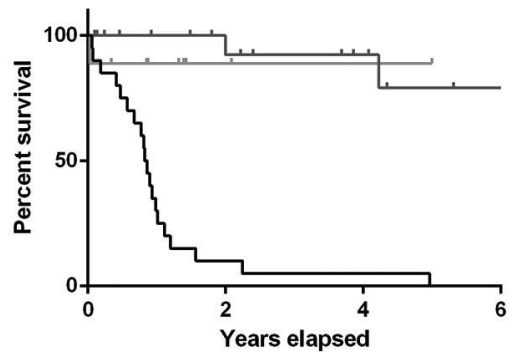

$$
\begin{aligned}
& \text { - Not Transplanted } \\
& \text { - Survival following } \\
& \text { transplant (age }<65 \text { ) } \\
& \text { Survival following }
\end{aligned}
$$

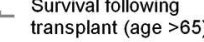

Abstract P203 Figure 1.

Conclusion As patients who suffer from IPF commonly present in their 7 th decade, the consideration of patient age is pertinent when referring for transplantation. Although, many centres view age greater than 65 as a relative contraindication to lung transplantation, we feel that this data reinforces the alternative viewpoint - that age should not be a limiting factor, in carefully selected candidates.

P204 REDUCING ANTIGEN EXPOSURE IN PIGEON BREEDERS. WEARING A MASK IS A SIGN OF DISEASE
${ }^{1} \mathrm{~K}$ McHugh, ${ }^{2}$ I Fraser, ${ }^{3} \mathrm{~S}$ Bourke, ${ }^{2} \mathrm{C}$ McSharry, ${ }^{1} \mathrm{P}$ Lynch, ${ }^{1} \mathrm{~K}$ Anderson; ${ }^{1}$ University Hospital Crosshouse, Kilmarnock, Scotland; '2Department of Immunolgy - Glasgow University, Glasgow, Scotland; ${ }^{3}$ Royal Victoria Infirmary, Newcastle, Scotland

\subsection{6/thoraxjnl-2013-204457.356}

Advice to pigeon breeders from the British Pigeon Fanciers Medical Research website includes methods to reduce dust exposure: as well as increasing loft ventilation, it recommends that a respirator is worn while in the loft. A field survey of the use of masks for this purpose found up to $40 \%$ usage amongst breeders $(\mathrm{n}=258)$. Studies in small numbers of HP patients demonstrated reducing symptom response following inhalation provocation tests as well as reducing serum antibody levels (1). Despite the potential benefit of wearing masks, and the apparent willingness of pigeon fanciers to do this, little is known about their protective value in HP.

The aim of this study is to compare survey results since 1991, and to examine the relationship between diagnosis of pigeon breeder's disease (PBD), serology, spirometry and mask wearing. Our 1997 survey $(\mathrm{n}=252)$ showed that $51 \%$ would use a mask. In our 2013 survey $(\mathrm{n}=188)$, we had a response of $54 \%$. Those wearing a mask have higher $\mathrm{IgG}(\mathrm{mcg} / \mathrm{ml})$ antibody levels (interquartile range 0.92-16.33 median 4.96 -v- 3.0217.04 median 8.55, $\mathrm{p}=0.047$ ). Questionnaire symptom responses, spirometry, as well as useful radiology (CT scanning) or biopsy were used to grade the likelihood of PBD as unlikely $(\mathrm{n}=99)$, maybe (57) or likely (32). Mask wearing was associated with likelihood of PBD $(\mathrm{p}=0.068)$. Those with symptoms ( $\mathrm{n}=46$ ) or minimal symptoms $(\mathrm{n}=38)$ performed spirometry. Analysis showed an apparent trend amongst subjects with a restrictive defect to be more marked amongst mask wearers$70 \%(\mathrm{n}=16)$ being moderate/severely restricted-compared to $43 \%$ non mask wearers (Chi-sq $=2.62, \mathrm{p}=0.105)$.

We interpret these findings as evidence that mask wearing is much more likely after symptoms have appeared in pigeon breeders: not the pattern we expected of mask wearing (or dust avoidance) to prevent the development of the disease. Paradoxically, mask wearing in pigeon breeders may also be an unreported sign of PBD.

\section{REFERENCES}

1. Hendrick, D J (1981) Protective value of dust respirator in extrinsic alveolitis: clinical assessment using inhaltion provocation tests. Thorax. 36: 917-921

\section{P205 21 YEARS OF SHIELD: DECREASING INCIDENCE OF OCCUPATIONAL ASTHMA IN THE WEST MIDLANDS, UK?}

${ }^{1} \mathrm{Gl}$ Walters, ${ }^{2} \mathrm{~A}$ Kirkham, ${ }^{1} \mathrm{EE}$ McGrath, ${ }^{1} \mathrm{VC}$ Moore, ${ }^{1} \mathrm{AS}$ Robertson, ${ }^{1} \mathrm{PS}$ Burge; ${ }^{1}$ Birmingham Heartlands Hospital, Birmingham, UK; ${ }^{2}$ University of Birmingham, Birmingham, UK

\subsection{6/thoraxjnl-2013-204457.357}

Introduction Notifications of occupational asthma (OA) to the West Midlands SHIELD reporting scheme have declined between 1991 and 2011. This may be due in part to reporter fatigue or restrictions on reporting, under-recognition, or true reduction in incidence of $\mathrm{OA}$ due to workplace control measures. We aimed to describe trends in reports of OA to the SHIELD database over a 21-year period and investigate reasons for any changes. Methods Descriptive statistics were performed on demographic and annual notification count data (for total notifications and individual causative agents). Count data were scaled to give a count per million workers using West Midlands' mid-year 
population estimates. A number of statistical analyses were undertaken to calculate trends in annual notifications: (i) nonparametric Kendall tau-b correlation (reference method), (ii) a negative binomial regression model, calculating incident rate ratios (IRRs), including a step-change analysis, and (iii) a logistic regression model calculating annual reporting odds ratios for 13 common causative agents.

Results 1637 cases of OA were notified between 1991 and 2011. Most cases were notified from the Birmingham Heartlands specialist clinic and notifications made elsewhere in the West Midlands fell from 16 per million workers in 1995 to zero in 2004, with very few cases (4 per million workers) after that. A significant non-linear decrease in annual total notifications was observed over the study period (IRR $=1.056 ; 95 \%$ $\mathrm{CI}=1.012-1.102 ; \mathrm{p}=0.012)$, as was the case for most causative agents studied. However cleaning products showed a $6 \%$ increase in notifications year-on-year (IRR $=1.056$; 95\% $\mathrm{CI}=1.012-1.102 ; \mathrm{p}=0.012$; Figure 1). Although the incidence of isocyanate-related OA decreased significantly after 2005 , the proportion of annual notifications due to isocyanates relative to other agents increased year on year ( $\operatorname{tau}=0.43$; $\mathrm{p}=0.007)$.

Conclusions Falling incidence of OA between 1991 and 2011 is explained in part by under-recognition by healthcare professionals, and by reporter fatigue, though for some causative agents this may be attributed in part to better workplace controls. Isocyanates remain the most common cause of OA but reports have fallen recently along with colophony, latex and glutaraldehyde, which have almost disappeared. Conversely cleaning product related OA is increasingly recognised.

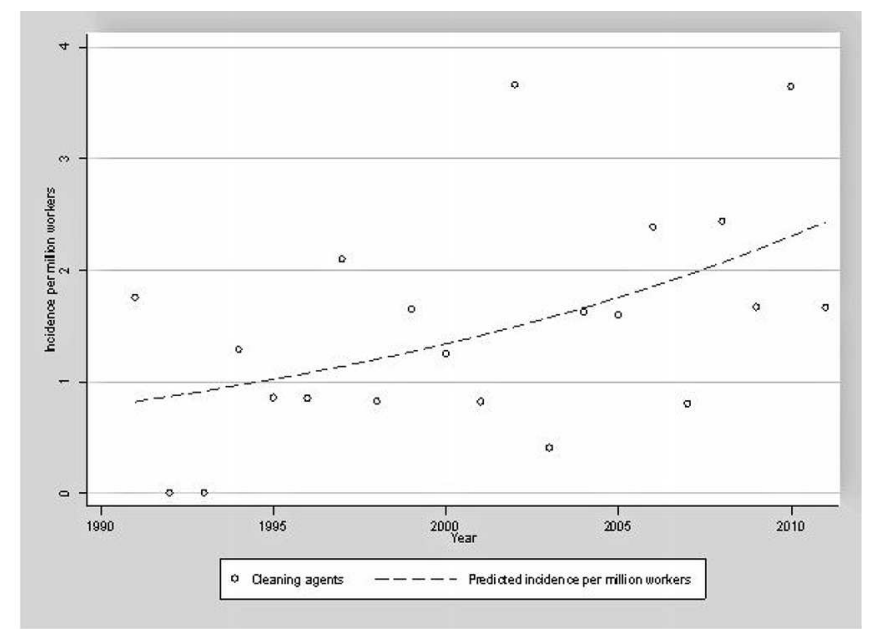

Abstract P205 Figure 1. Annual notifications of occupational asthma due to cleaning products (cases per million workers) recorded 1991-2011 in West Midlands UK, with prediction line based on negative binomial regression analysis.

\section{P206 ASBESTOS RELATED DIFFUSE PLEURAL THICKENING; LIKELIHOOD OF PROGRESSION IN THE SECONDARY CARE SETTING}

JL Hoyle, S Gudur; North Manchester General Hospital, Manchester, UK

\subsection{6/thoraxjnl-2013-204457.358}

Introduction There is a lack of recent evidence in terms of progression and prognosis in subjects with asbestos induced diffuse pleural thickening (DPT). We reviewed those with a diagnosis of DPT to look at radiological progression and prognosis.

Methods A local database search identified those registered with DPT as agreed by a Multidisciplinary Meeting (MDT) and defined by Industrial Injuries Disablement Benefit (IIDB) as including obliteration of a costophrenic angle on chest $\mathrm{x}$-ray (CXR) and mesothelioma previously ruled out. Years of followup and progression on radiology defined as a measureable increase in thickening unilaterally on CXR, or new obliteration of a costophrenic angle contralaterally. All causes of death were recorded.

Results 39 of 48 subjects registered, all male had local follow up. Radiological follow-up occurred for between 1 and 10 years, mean 3.9 yrs.

In total $16 / 39(41 \%)$ had radiological progression on CXR. 7 $(18 \%)$ died, 6/39 (15\%) from respiratory related illness. 6/39 had coexistent asbestosis.

28/39 (72\%) presented with unilateral DPT of whom 5/28 (18\%) had asbestosis on CT.

11/28 (39\%) progressed from unilateral to bilateral DPT including 3 with asbestosis, 4/28 (14\%) died, (1 with DPT and asbestosis developed mesothelioma), 1 of non-respiratory cancer, 2 died respiratory failure; however 35\% (8/23) of the unilateral DPT only cohort also progressed or died.

$5 / 11(45 \%)$ presenting with bilateral DPT progressed or died. 2 (18\%) increased thickening on CXR. 3/11 (27\%) died, 1 lung cancer (with asbestosis) and 2 respiratory failure.

Discussion Although DPT is considered benign, 41\% progressed including 39\% with unilateral DPT who developed bilateral disease. $15 \%$ had coexistent asbestosis and as expected were more likely to progress or die. $11 \%$ of those presenting with unilateral disease on CXR and over 27\% with bilateral disease died of respiratory cause over the follow-up period.

Conclusions Not withstanding the limitations of this study, the likelihood of radiological progression with a diagnosis of DPT is 2 in 5 , including those with unilateral disease. The risk of death from a respiratory cause with bilateral DPT diagnosed on CXR (27\%) is more than twice that for unilateral disease on CXR (11\%).

\section{Abstract P206 Table 1.

\begin{tabular}{|c|c|}
\hline $\begin{array}{l}\text { Unilateral DPT on CXR } \\
N=28\end{array}$ & $\begin{array}{l}\text { Bilateral DPT on CXR } \\
\mathrm{N}=11\end{array}$ \\
\hline CT & CT \\
\hline
\end{tabular}

Progression DPT only Asbestosis seen DPT only Asbestosis seen Total

\begin{tabular}{llllll} 
Stable & 15 & 2 & 6 & 0 & 23 \\
Progressed & 5 & 2 & 2 & 0 & 9 \\
Died & $3^{*}$ & 1 & 2 & 1 & 7 \\
Total & 23 & 5 & 10 & 1 & 39 \\
\hline
\end{tabular}

*1 died of non-respiratory cancer

CT-Computed Tomography of lungs

DPT-Diffuse pleural thickening

\section{P207 REPORTING OF ASBESTOS RELATED LUNG DISEASE TO HM CORONER: A RETROSPECTIVE ANALYSIS}

C Waterworth, G Hughes, B Srinivasan, BG Marshall; Southampton General Hospital, Southampton, UK

\subsection{6/thoraxjnl-2013-204457.359}

Background Asbestos related lung disease consists of a spectrum of disorders, ranging from benign pleural plaques to 